\title{
SOME NEW GENERALIZATIONS OF HARDY'S INTEGRAL INEQUALITY
}

\author{
S. K. SUNANDA, C. NAHAK, AND S. NANDA
}

Received 5 March 2006; Revised 31 May 2006; Accepted 5 June 2006

We have studied some new generalizations of Hardy's integral inequality using the generalized Holder's inequality.

Copyright (c) 2006 Hindawi Publishing Corporation. All rights reserved.

\section{Introduction}

The classical Hardy's inequality [2] states that for $p>1,1 / p+1 / q=1, f \geq 0$, and $0<$ $\int_{0}^{\infty} f^{p}(t) d t<\infty$, then

$$
\int_{0}^{\infty}\left[\frac{1}{x} \int_{0}^{x} f(t) d t\right]^{p} d x<q^{p} \int_{0}^{\infty} f^{p}(t) d t
$$

where $q^{p}=(p /(p-1))^{p}$ is the best possible. This inequality plays important role in analysis. It is obvious that, for parameters $a$ and $b$ such that $0<a<b<\infty$, the following inequality is also valid:

$$
\int_{a}^{b}\left[\frac{1}{x} \int_{a}^{x} f(t) d t\right]^{p} d x<q^{p} \int_{a}^{b} f^{p}(t) d t
$$

where $0<\int_{a}^{b} f^{P}(t) d t<\infty$.

Bicheng et al. [1] have given some improvements of (1.1) and (1.2) as follows.

Let $0<a<b<\infty, p>1,1 / p+1 / q=1, f \geq 0$, and $0<\int_{a}^{b} f^{p}(t) d t<\infty$, then

$$
\begin{aligned}
\int_{a}^{b}\left[\frac{1}{x} \int_{a}^{x} f(t) d t\right]^{p} d x \\
\quad<q^{p}\left[1-\left(\frac{a}{b}\right)^{1 / q}\right]^{p} \int_{a}^{b} f^{p}(t) d t .
\end{aligned}
$$

Hindawi Publishing Corporation

International Journal of Mathematics and Mathematical Sciences

Volume 2006, Article ID 19013, Pages 1-15

DOI 10.1155/IJMMS/2006/19013 
2 Some new generalizations of Hardy's integral inequality

Let $a>0, p>1,1 / p+1 / q=1, f \geq 0$, and $0<\int_{a}^{\infty} f^{p}(t) d t<\infty$, then

$$
\int_{a}^{\infty}\left[\frac{1}{x} \int_{a}^{x} f(t) d t\right]^{p} d x<q^{p} \int_{a}^{\infty}\left[1-\theta_{p}(t)\right] f^{p}(t) d t
$$

where $\theta_{p}(t)=1 / p \sum_{k=1}^{\infty}\left(\begin{array}{l}p \\ k\end{array}\right)(-1)^{k-1}(a / t)^{k / q}>0$ for $t>a$, and $\theta_{p}(a)=1 / q$.

Oguntuase and Imoru [3] generalized (1.3) and (1.4) as follows.

Let $0<a<b<\infty, p>1,1 / p+1 / q=1-1 / r, f \geq 0, r>1$, and $0<\int_{a}^{b} f^{p}(t) d t<\infty$, then

$$
\begin{aligned}
\int_{a}^{b}[ & \left.\frac{1}{x^{(1-1 / r)}} \int_{a}^{x} f(t) d t\right]^{p} d x \\
& <q^{(1-1 / r) p}\left(1-\frac{1}{r}\right)^{(1-1 / r) p}\left[1-\left(\frac{a}{b}\right)^{1 / q}\right]^{(1-1 / r) p} \int_{a}^{b} f^{p}(t) d t .
\end{aligned}
$$

Let $a>0, p>1,1 / p+1 / q=1-1 / r, f \geq 0, r>1$, and $0<\int_{a}^{\infty} f^{p}(t) d t<\infty$, then

$$
\int_{a}^{\infty}\left[\frac{1}{x^{(1-1 / r)}} \int_{a}^{x} f(t) d t\right]^{p} d x<q^{(1-1 / r) p}\left(1-\frac{1}{r}\right)^{(1-1 / r) p} \int_{a}^{\infty}\left[1-\theta_{p}(t)\right] f^{p}(t) d t
$$

where $\theta_{p}(t)=1 /(1-1 / r) p \sum_{k=1}^{\infty}\left(\begin{array}{c}(1-1 / r) p \\ k+1\end{array}\right)(-1)^{k-1}(a / t)^{k /(1-1 / r) q}>0$ for $t>a$, and $\theta_{p}(a)=$ $1 /(1-1 / r) q$.

Definition 1.1. Let $1 \leq p<\infty$, then the function space $L_{p}$ is given by

$$
L_{p}=\left\{f: \int_{0}^{\infty}|f(x)|^{p} d x<\infty\right\}
$$

The function space $L_{p}$ has been generalized to $L(p)$ in the following manner.

Definition 1.2. Let $p$ be a bounded measurable function, with $0<p(x) \leq \sup p(x)=H<$ $\infty$. Define

$$
L(p)=\left\{f: \int_{0}^{\infty}|f(x)|^{p(x)} d x<\infty\right\}
$$

Note that $L(p)$ is a linear topological space paranormed by $d(f)$,

$$
d(f)=\left(\int_{0}^{\infty}|f(x)|^{p(x)} d x\right)^{1 / M}
$$

where $M=\max (1, H)$.

In this paper, we have the generalized Holder's inequality in $L(p)$ space and the results of $[1,3]$. 


\section{Main results}

LEMma 2.1. (a) Let the functions $p$ and $q$ be such that $p(x)^{-1}+q(x)^{-1}=1$ for all $x$. Let $f \in L(p), g \in L(q)$. Let

$$
A=\int_{0}^{\infty}|f(x)|^{p(x)} d x, \quad B=\int_{0}^{\infty}|g(x)|^{q(x)} d x .
$$

Then for $p(x)>1, f g \in L_{1}$ and

$$
\int_{0}^{\infty}|f(x) g(x)| d x \leq \alpha \beta
$$

where $\alpha=\sup _{x} 1 / p(x)+\sup _{x} 1 / q(x), \beta=\sup _{x} A^{1 / p(x)} B^{1 / q(x)}$.

(b) Let $0<p(x)<1$ and $p(x)^{-1}+q(x)^{-1}=1$. If $f \in L(p)$ and $g \in L(q)$, then

$$
\int_{0}^{\infty}|f(x)|^{p(x)} d x \leq \alpha\left[\sup _{x} p(x)+\sup _{x}(1-p(x))\right]
$$

where $\alpha=\sup _{x}\left[\left(\int|g(x)|^{q(x)} d x\right)^{1-p(x)}\left(\int|f(x) g(x)| d x\right)^{p(x)}\right]$.

Proof. To prove (a), for $a, b>0$, we have

$$
a b \leq \frac{a^{p(x)}}{p(x)}+\frac{b^{q(x)}}{q(x)} \quad \forall x .
$$

Using the above inequality, we have

$$
\frac{|f(x)|}{A^{1 / p(x)}} \frac{|g(x)|}{B^{1 / q(x)}} \leq \frac{1}{p(x)} \frac{|f(x)|^{p(x)}}{A}+\frac{1}{q(x)} \frac{|g(x)|^{q(x)}}{B} .
$$

Therefore,

$$
\begin{aligned}
\int_{0}^{\infty} \frac{|f(x)||g(x)|}{A^{1 / p(x)} B^{1 / q(x)}} d x & \leq \frac{1}{A} \int_{0}^{\infty} \frac{|f(x)|^{p(x)}}{p(x)} d x+\frac{1}{B} \int_{0}^{\infty} \frac{|g(x)|^{q(x)}}{q(x)} d x \\
& \leq \sup _{x} \frac{1}{p(x)}+\sup _{x} \frac{1}{q(x)} .
\end{aligned}
$$

Also

$$
\frac{1}{\sup _{x} A^{1 / p^{(x)}} B^{1 / q(x)}} \int_{0}^{\infty}|f(x) g(x)| d x \leq \int_{0}^{\infty} \frac{|f(x) g(x)|}{A^{1 / p(x)} B^{1 / q(x)}} d x
$$

From (2.6) and (2.7), we get (2.2).

To prove (b), let $p_{1}(x)=1 / p(x)$, so that $p_{1}(x)>1$ for all $x$. Let

$$
A(x)=|g(x)|^{-1 / p_{1}(x)}, \quad B(x)=|f(x) g(x)|^{1 / p_{1}(x)} .
$$


4 Some new generalizations of Hardy's integral inequality

So

$$
\begin{aligned}
\int_{0}^{\infty}|f(x)|^{p(x)} d x= & \int_{0}^{\infty}|A(x) B(x)| d x \\
\leq & \sup _{x}\left[\left(\int_{0}^{\infty}|A(x)|^{q_{1}(x)} d x\right)^{1 / q_{1}(x)}\left(\int_{0}^{\infty}|B(x)|^{p_{1}(x)} d x\right)^{1 / p_{1}(x)}\right] \\
& \times\left(\sup _{x} \frac{1}{p_{1}(x)}+\sup _{x} \frac{1}{q_{1}(x)}\right) \quad \text { by }(2.2) .
\end{aligned}
$$

Since $1 / p_{1}(x)+1 / q_{1}(x)=1$, so $1 / q_{1}(x)=1-p(x)$ and $q_{1}(x)=1 / 1-p(x)$.

Substituting the values, we get $(2.3)$ :

$$
\begin{aligned}
\int_{0}^{\infty}|f(x)|^{p(x)} d x= & \sup _{x}\left[\left(\int_{0}^{\infty}|g(x)|^{q(x)} d x\right)^{1-p(x)}\left(\int_{0}^{\infty}|f(x) g(x)| d x\right)^{p(x)}\right] \\
& \times\left[\sup _{x} p(x)+\sup _{x}(1-p(x))\right]=\alpha\left[\sup _{x} p(x)+\sup _{x}(1-p(x))\right] .
\end{aligned}
$$

This completes the proof of the lemma.

Lemma 2.2. Let $0<b \leq \infty$, for all $x \in(0, b), p(x)>1, p(x)^{-1}+q(x)^{-1}=1, f \geq 0$, and $0<\int_{0}^{b} f^{p(t)}(t) d t<\infty$. Then the following inequality holds:

$$
\int_{0}^{x} f(t) d t<\alpha \sup _{x \in(0, b)}\left\{\{\inf q(x)\}^{1 / q(x)} x^{\sup 1 / q(x) 2}\left[\int_{0}^{x} t^{1 / q(t)} f^{p(t)}(t) d t\right]^{1 / p(x)}\right\}
$$

where $\alpha=\sup _{x} 1 / p(x)+\sup _{x} 1 / q(x)$.

Proof. For any $x \in(0, b)$, by the generalized Holder's inequality (2.2), we have

$$
\begin{aligned}
\int_{0}^{x} f(t) d t & =\int_{0}^{x} t^{1 / p(t) q(t)} f(t) t^{-1 / p(t) q(t)} d t \\
& \leq \alpha \sup _{x \in(0, b)}\left\{\left(\int_{0}^{x} t^{1 / q(t)} f^{p(t)}(t) d t\right)^{1 / p(x)}\left(\int_{0}^{x} t^{-1 / p(t)} d t\right)^{1 / q(x)}\right\} \\
& =\alpha \sup _{x \in(0, b)}\left\{\{\inf q(x)\}^{1 / q(x)} x^{\sup 1 / q(x) 2}\left[\int_{0}^{x} t^{1 / q(t)} f^{p(t)}(t) d t\right]^{1 / p(x)}\right\} .
\end{aligned}
$$

Strictness follows from [1, Lemma 2.1]. Thus (2.11) is valid.

Lemma 2.3. Let $a \geq 0$, for all $x \in(a, \infty), p(x)>1, p(x)^{-1}+q(x)^{-1}=1, f \geq 0$, and $0<$ $\int_{a}^{x} f^{p(t)}(t) d t<\infty$. Then the following inequality is true:

$$
\int_{a}^{x} f(t) d t<\alpha \sup _{x \in(a, \infty)}\left\{\{\inf q(x)\}^{1 / q(x)}\left(x^{\sup 1 / q(x)}-a^{\sup 1 / q(x)}\right)\left[\int_{a}^{x} t^{1 / q(t)} f^{p(t)}(t) d t\right]^{1 / p(x)}\right\},
$$

where $\alpha=\sup _{x} 1 / p(x)+\sup _{x} 1 / q(x)$. 
Proof. For any $x \in(a, \infty)$, by the generalized Holder's inequality (2.2), we have

$$
\begin{aligned}
\int_{a}^{x} f(t) d t & \leq \alpha \sup _{x \in(a, \infty)}\left\{\left(\int_{a}^{x} t^{1 / q(t)} f^{p(t)}(t) d t\right)^{1 / p(x)}\left(\int_{a}^{x} t^{-1 / p(t)} d t\right)^{1 / q(x)}\right\} \\
& \leq \alpha \sup _{x \in(a, \infty)}\left\{\{\inf q(x)\}^{1 / q(x)}\left(x^{\sup 1 / q(x)}-a^{\sup 1 / q(x)}\right)\left[\int_{a}^{x} t^{1 / q(t)} f^{p(t)}(t) d t\right]^{1 / p(x)}\right\} .
\end{aligned}
$$

Strictness follows from [1, Lemma 2.2]. This completes the proof of the lemma.

Theorem 2.4. Let $0<a<b<\infty$, for all $x \in(a, b), p(x)>1, p(x)^{-1}+q(x)^{-1}=1, f \geq 0$, and $0<\int_{a}^{b} f^{p(t)}(t) d t<\infty$. Then

$$
\int_{a}^{b}\left(\frac{1}{x} \int_{a}^{x} f(t) d t\right)^{p(x)} d x<\alpha^{M}\{\inf q(t)\}^{M}\left[1-\left(\frac{a}{b}\right)^{\sup 1 / q(t)}\right]^{M} \int_{a}^{b} f^{p(t)}(t) d t,
$$

where $M=\sup p(t), \alpha=\sup \left\{p(t)^{-1}\right\}+\sup \left\{q(t)^{-1}\right\}$.

Proof. Using (2.13), we obtain

$$
\begin{aligned}
& \int_{a}^{b}\left(\frac{1}{x} \int_{a}^{x} f(t) d t\right)^{p(x)} d x \\
&< \alpha^{\sup p(x)} \int_{a}^{b} x^{-p(x)}\left(x^{\sup 1 / q(x)}-a^{\sup 1 / q(x)}\right)^{p(x) / q(x)}\{\inf q(x)\}^{p(x) / q(x)} \\
& \times \int_{a}^{x} t^{1 / q(t)} f^{p(t)}(t) d t d x \leq \alpha^{\sup p(t)}\{\inf q(t)\}^{\sup p(t) / q(t)} \\
& \times \int_{a}^{b}\left\{\int_{t}^{b} x^{-p(x)+\sup \left(p(x) / q(x)^{2}\right)}\left(1-\left(\frac{a}{x}\right)^{\sup (1 / q(x))}\right)^{p(x) / q(x)} d x\right\} t^{1 / q(t)} f^{p(t)}(t) d t \\
& \leq \alpha^{M}\{\inf q(t)\}^{\sup (p(t)-1)} \\
& \times \int_{a}^{b}\left\{\int_{t}^{b} x^{-1-1 / q(x)}\left(1-\left(\frac{a}{b}\right)^{\sup 1 / q(x)}\right)^{p(x) / q(x)} d x\right\} t^{1 / q(t)} f^{p(t)}(t) d t \\
& \leq \alpha^{M}\{\inf q(t)\}^{\sup p(t)}\left[1-\left(\frac{a}{b}\right)^{\sup (1 / q(t))}\right]^{\sup p(t)-1} \int_{a}^{b}\left[1-\left(\frac{t}{b}\right)^{\sup (1 / q(t))}\right] f^{p(t)}(t) d t \\
& \leq \alpha^{M}\{\inf q(t)\}^{M}\left[1-\left(\frac{a}{b}\right)^{\sup (1 / q(t))}\right]^{M} \int_{a}^{b} f^{p(t)}(t) d t .
\end{aligned}
$$

Theorem 2.5. Let $a>0$, for all $x \in(a, \infty), p(x)>1, p(x)^{-1}+q(x)^{-1}=1, f \geq 0$, and $0<$ $\int_{a}^{\infty} f^{p(t)}(t) d t<\infty$. Then

$$
\int_{a}^{\infty}\left(\frac{1}{x} \int_{a}^{x} f(t) d t\right)^{p(x)} d x<\alpha^{M}\{\inf q(t)\}^{M} \int_{a}^{\infty}\left[1-\theta_{M}(t)\right] f^{p(t)}(t) d t,
$$


6 Some new generalizations of Hardy's integral inequality

where $\theta_{M}(t)=(1 / M)\left[\sum_{k=1}^{\infty}(\Gamma(M+1) / \Gamma(k+2) \Gamma(M-k))(-1)^{k-1}(a / t)^{(k / \inf q(t))}\right]$, for $t>a>$ $0, M>1$, and $\theta_{M}(a)=\sup 1 / q(t), M=\sup p(t)$, and $\alpha=\sup \left\{p(t)^{-1}\right\}+\sup \left\{q(t)^{-1}\right\}$.

Proof. In view of inequalities (2.13) and (2.15), we find

$$
\begin{aligned}
& \int_{a}^{\infty}\left(\frac{1}{x} \int_{a}^{x} f(t) d t\right)^{p(x)} d x \\
& <\alpha^{\sup p(x)} \int_{a}^{\infty} x^{-p(x)}\left(x^{\sup 1 / q(x)}-a^{\sup 1 / q(x)}\right)^{p(x) / q(x)}\{\inf q(x)\}^{p(x) / q(x)} \\
& \times \int_{a}^{x} t^{1 / q(t)} f^{p(t)}(t) d t d x \leq \alpha^{\sup p(t)}\{\inf q(t)\}^{\sup (p(t)-1)} \\
& \times \int_{a}^{\infty}\left\{\int_{t}^{\infty} x^{-1-1 / q(x)}\left(1-\left(\frac{a}{b}\right)^{\sup (1 / q(x))}\right)^{p(x) / q(x)} d x\right\} t^{1 / q(t)} f^{p(t)}(t) d t \\
& \leq \alpha^{M}\{\inf q(t)\}^{\sup (p(t)-1)} \\
& \times \int_{a}^{\infty}\left[\int_{t}^{\infty}\left(1-\left(\frac{a}{b}\right)^{\sup (1 / q(x))}\right)^{p(x) / q(x)} d\left(1-\left(\frac{a}{b}\right)^{\sup (1 / q(x))}\right)\right] \\
& \times\left(\frac{t}{a}\right)^{\sup (1 / q(t))} f^{p(t)}(t) d t \leq \alpha^{M}\{\inf q(t)\}^{\sup p(t)} \\
& \times \int_{a}^{\infty} \frac{1}{\sup p(t)}\left\{1-\left[1-\left(\frac{a}{t}\right)^{\sup (1 / q(t))}\right]^{\sup p(t)}\right\}\left(\frac{t}{a}\right)^{\sup (1 / q(t))} f^{p(t)}(t) d t \\
& =\alpha^{M}\{\inf q(t)\}^{M} \int_{a}^{\infty}\left[1-\theta_{M}(t)\right] f^{p(t)}(t) d t,
\end{aligned}
$$

where

$$
\begin{gathered}
\theta_{M}(t)=1-\frac{1}{M}\left\{1-\left[1-\left(\frac{a}{t}\right)^{\sup (1 / q(t))}\right]^{M}\right\}\left(\frac{t}{a}\right)^{\sup (1 / q(t))}, t>a>0, M>1, \\
\theta_{M}(a)=\sup \frac{1}{q(t)} .
\end{gathered}
$$

Since

$$
\begin{gathered}
{\left[1-\left(\frac{a}{t}\right)^{\sup (1 / q(t))}\right]^{M}=\sum_{k=0}^{\infty} \frac{\Gamma(M+1)}{\Gamma(k+1) \Gamma(M-k+1)}(-1)^{k}\left(\frac{a}{t}\right)^{k / \inf q(t)}, \quad t>a>0, M>1,} \\
\theta_{M}(t)=\frac{1}{M}\left[\sum_{k=1}^{\infty} \frac{\Gamma(M+1)}{\Gamma(k+2) \Gamma(M-k)}(-1)^{k-1}\left(\frac{a}{t}\right)^{k / \inf q(t)}\right], \quad t>a>0, M>1,
\end{gathered}
$$

the proof is complete. 
Note. When $t>a>0$, by Bernoulli's inequality (see [2, Chapter 2.4]), we obtain

$$
\begin{gathered}
1-M\left(\frac{a}{t}\right)^{\sup 1 / q(t)}<\left[1-\left(\frac{a}{t}\right)^{\sup 1 / q(t)}\right]^{M}, \\
\theta_{M}(t)>1-\frac{1}{M}\left[1-\left\{1-M\left(\frac{a}{t}\right)^{\sup 1 / q(t)}\right\}\right]\left(\frac{t}{a}\right)^{\sup 1 / q(t)}=0 .
\end{gathered}
$$

\section{Applications}

Theorem 2.6. Let $0<b \leq \infty$, for all $x \in(0, \infty), r \geq p(x)>1, p(x)^{-1}+q(x)^{-1}=1, f \geq 0$, and $0<\int_{0}^{b} x^{-r+\sup p(x)} f^{p(x)}(x)<\infty$. Then

(i) for $b \in(0, \infty)$,

$$
\begin{aligned}
\int_{0}^{b} x^{-r} & \left(\int_{0}^{x} f(t) d t\right)^{p(x)} d x \\
& <\frac{\alpha^{M}\{\inf q(t)\}^{M}}{\inf \{(r-p(t)) q(t)+1\}} \int_{0}^{b}\left[1-\left(\frac{t}{b}\right)^{\sup \{r-p(t)+1 / q(t)\}}\right] t^{-r+\sup p(t)} f^{p(t)}(t) d t
\end{aligned}
$$

(ii) for $b=\infty$,

$$
\int_{0}^{\infty} x^{-r}\left(\int_{0}^{x} f(t) d t\right)^{p(x)} d x<\frac{\alpha^{M}\{\inf q(t)\}^{M}}{\inf \{(r-p(t)) q(t)+1\}} \int_{0}^{\infty} t^{-r+\sup p(t)} f^{p(t)}(t) d t
$$

where $M=\sup p(t)$ and $\alpha=\sup \left\{p(t)^{-1}\right\}+\sup \left\{q(t)^{-1}\right\}$.

Proof. For case (i), $b \in(0, \infty)$, we use $(2.11)$ to obtain

$$
\begin{aligned}
& \int_{0}^{b} x^{-r}\left(\int_{0}^{x} f(t) d t\right)^{p(x)} d x \\
& \quad<\alpha^{M} \int_{0}^{b} x^{-r+\sup \{p(x) / q(x) 2\}}\{\inf q(x)\}^{p(x) / q(x)} \int_{0}^{x} t^{1 / q(t)} f^{p(t)}(t) d t d x \\
& \quad \leq \alpha^{M}\{\inf q(t)\}^{\sup \{p(t)-1\}} \int_{0}^{b}\left(\int_{t}^{b} x^{-r+\sup \{p(x)-1-1 / q(x)\}} d x\right) t^{1 / q(t)} f^{p(t)}(t) d t \\
& \quad \leq \frac{\alpha^{M}\{\inf q(t)\}^{M}}{-r+\sup \{p(t)-1 / q(t)\}} \int_{0}^{b}\left(b^{-r+\sup \{p(t)-1 / q(t)\}}-t^{-r+\sup \{p(t)-1 / q(t)\}}\right) \\
& \quad \leq \frac{\alpha^{M}\{\inf q(t)\}^{M}}{\inf \{(r-p(t)) q(t)+1\}} \int_{0}^{b}\left[1-\left(\frac{t}{b}\right)^{\sup \{r-p(t)+1 / q(t)\}}\right] t^{-r+\sup p(t)} f^{p(t)}(t) d t .
\end{aligned}
$$


8 Some new generalizations of Hardy's integral inequality

For case (ii), $b=\infty$, we use (2.11) to find

$$
\begin{aligned}
\int_{0}^{\infty} x^{-r} & \left(\int_{0}^{x} f(t) d t\right)^{p(x)} d x \\
& <\alpha^{M}\{\inf q(t)\}^{\sup (p(t)-1)} \int_{0}^{\infty} x^{-r+\sup \left\{p(x) / q(x)^{2}\right\}} \int_{0}^{x} t^{1 / q(t)} f^{p(t)}(t) d t d x \\
& =\alpha^{M}\{\inf q(t)\}^{\sup (p(t)-1)} \int_{0}^{\infty}\left(\int_{t}^{\infty} x^{-r+\sup \{p(x)-1-1 / q(x)\}} d x\right) t^{1 / q(t)} f^{p(t)}(t) d t \\
& =\frac{\alpha^{M}\{\inf q(t)\}^{M}}{\inf \{(r-p(t)) q(t)+1\}} \int_{0}^{\infty} t^{-r+\sup \{p(t)\}} f^{p(t)}(t) d t .
\end{aligned}
$$

Remark 2.7. (a) If $p(x)$ and $q(x)$ are constants in Lemma 2.1, then (1.6) reduces to usual Holder's inequality in $L_{p}$ space.

(b) If we take $p(x)$ and $q(x)$ constants in Lemmas 2.2 and 2.3 and Theorems 2.4 and 2.5, then our results reduce to the corresponding Lemmas 2.1 and 2.2 and Theorems 2.4 and 2.5 obtained in [1].

(c) When $p(x)=r$ and $q(x)$ is constant, inequality (2.23) reduces to (1.1).

\section{Some more generalized results}

In this section, we have generalized the results of [3]. We use the generalized form of Holder's inequality with $p(x), q(x), r(x)>1$. The normalization $1 / p(x)+1 / q(x)=1$ in Holder's inequality is replaced by relation of the form $1 / p(x)+1 / q(x)=1-1 / r(x)$.

Lemma 3.1. Let $0<b \leq \infty$, for all $x \in(0, b), p(x)>1, p(x)^{-1}+q(x)^{-1}=1-r(x)^{-1}, f \geq 0$, $r(x)>1$, and $0<\int_{0}^{b} f^{p(t)}(t) d t<\infty$. Then the following inequality holds:

$$
\begin{aligned}
\int_{0}^{x} f(t) d t<\alpha \sup _{x \in(0, b)} & \left\{\left\{\inf \left\{\left(1-\frac{1}{r(x)}\right) q(x)\right\}\right\}^{1 / q(x)} x^{\sup 1 /\left\{(1-1 / r(x)) q(x)^{2}\right\}}\right. \\
\times & {\left.\left[\int_{0}^{x} t^{1 /\{(1-1 / r(t)) q(t)\}} f^{p(t)}(t) d t\right]^{1 / p(x)}\right\}, }
\end{aligned}
$$

where $\alpha=\sup _{x} 1 / p(x)+\sup _{x} 1 / q(x)+\sup _{x} 1 / r(x)$.

Proof. For any $x \in(0, b)$, by the generalized Holder's inequality (2.2), we have

$$
\begin{aligned}
\int_{0}^{x} f(t) d t & =\int_{0}^{x} t^{1 /\{(1-1 / r(t)) p(t) q(t)\}} f(t) t^{-1 /\{(1-1 / r(t)) p(t) q(t)\}} d t \\
& \leq \alpha \sup _{x \in(0, b)}\left\{\left(\int_{0}^{x} t^{1 /\{(1-1 / r(t)) q(t)\}} f^{p(t)}(t) d t\right)^{1 / p(x)}\left(\int_{0}^{x} t^{-1 /\{(1-1 / r(t)) p(t)\}} d t\right)^{1 / q(x)}\right\}
\end{aligned}
$$


S. K. Sunanda et al. 9

$$
\begin{aligned}
\leq \alpha \sup _{x \in(0, b)}\{ & \left(\int_{0}^{x} t^{\sup \{-1 /\{(1-1 / r(t)) p(t)\}\}} d t\right)^{1 / q(x)} \\
\times & \left.\left(\int_{0}^{x} t^{1 /\{(1-1 / r(t)) q(t)\}} f^{p(t)}(t) d t\right)^{1 / p(x)}\right\} \\
=\alpha \sup _{x \in(0, b)}\{ & \left\{\inf \left\{\left(1-\frac{1}{r(x)}\right) q(x)\right\}\right\}^{1 / q(x)} x^{\sup 1 /\left\{(1-1 / r(x)) q(x)^{2}\right\}} \\
\times & {\left.\left[\int_{0}^{x} t^{\{1 /(1-1 / r(t)) q(t)\}} f^{p(t)}(t) d t\right]^{1 / p(x)}\right\} . }
\end{aligned}
$$

Strictness follows from [3, Lemma 2.1]. Thus (3.1) is valid.

Lemma 3.2. Let $a \geq 0$, for all $x \in(a, \infty), p(x)>1, p(x)^{-1}+q(x)^{-1}=1-r(x)^{-1}, f \geq 0$, $r(x)>1$, and $0<\int_{a}^{x} f^{p(t)}(t) d t<\infty$. Then the following inequality holds:

$$
\begin{aligned}
\int_{a}^{x} f(t) d t<\alpha \sup _{x \in(a, \infty)} & \left\{\left\{\inf \left\{\left(1-\frac{1}{r(x)}\right) q(x)\right\}\right\}^{1 / q(x)}\right. \\
& \times\left(x^{\sup 1 /\{(1-1 / r(x)) q(x)\}}-a^{\sup 1 /\{(1-1 / r(x)) q(x)\}}\right)^{1 / q(x)} \\
& \left.\times\left[\int_{a}^{x} t^{1 /\{(1-1 / r(t)) q(t)\}} f^{p(t)}(t) d t\right]^{1 / p(x)}\right\}
\end{aligned}
$$

where $\alpha=\sup _{x} 1 / p(x)+\sup _{x} 1 / q(x)+\sup _{x} 1 / r(x)$.

Proof. For any $x \in(a, \infty)$, by the generalized Holder's inequality (2.2), we have

$$
\begin{aligned}
\int_{a}^{x} f(t) d t \leq \alpha \sup _{x \in(a, \infty)}\{ & \left(\int_{a}^{x} t^{\sup \{-1 /\{(1-1 / r(t)) p(t)\}\}} d t\right)^{1 / q(x)} \\
& \left.\times\left(\int_{a}^{x} t^{1 /\{(1-1 / r(t)) q(t)\}} f^{p(t)}(t) d t\right)^{1 / p(x)}\right\} \\
\leq \alpha \sup _{x \in(a, \infty)}\{ & \left\{\inf \left\{\left(1-\frac{1}{r(x)}\right) q(x)\right\}\right\}^{1 / q(x)} \\
& \times\left(x^{\sup 1 /\{(1-1 / r(x)) q(x)\}}-a^{\sup 1 /\{(1-1 / r(x)) q(x)\}}\right)^{1 / q(x)} \\
& \left.\times\left(\int_{a}^{x} t^{1 /\{(1-1 / r(t)) q(t)\}} f^{p(t)}(t) d t\right)^{1 / p(x)}\right\} .
\end{aligned}
$$

Strictness follows from [3, Lemma 2.2]. This completes the proof of the lemma. 
10 Some new generalizations of Hardy's integral inequality

Theorem 3.3. Let $0<a<b<\infty$, for all $x \in(a, b), p(x)>1, p(x)^{-1}+q(x)^{-1}=1-r(x)^{-1}$, $f \geq 0, r(x)>1$, and $0<\int_{a}^{b} f^{p(t)}(t) d t<\infty$. Then

$$
\begin{aligned}
& \int_{a}^{b}\left(\frac{1}{x^{(1-1 / r(x))}} \int_{a}^{x} f(t) d t\right)^{p(x)} d x \\
& \quad<\alpha^{M}\left\{\inf \left\{\left(1-\frac{1}{r(t)}\right) q(t)\right\}\right\}^{N}\left[1-\left(\frac{a}{b}\right)^{\sup 1 /\{(1-1 / r(t)) q(t)\}}\right]^{N} \int_{a}^{b} f^{p(t)}(t) d t,
\end{aligned}
$$

where $M=\sup p(t), \alpha=\sup 1 / p(t)+\sup 1 / q(t)+\sup 1 / r(t)$, and $N=\sup \{p(t)(1-1 /$ $r(t))\}$.

Proof. Using (3.3), we obtain

$$
\begin{aligned}
& \int_{a}^{b}\left(\frac{1}{x^{(1-1 / r(x))}} \int_{a}^{x} f(t) d t\right)^{p(x)} d x \\
& <\int_{a}^{b}\left[\frac { 1 } { x ^ { ( 1 - 1 / r ( x ) ) } } \alpha \operatorname { s u p } \left\{\left\{\inf \left\{\left(1-\frac{1}{r(x)}\right) q x\right\}\right\}^{1 / q(x)}\right.\right. \\
& \times\left(x^{\sup 1 /\{(1-1 / r(x)) q(x)\}}-a^{\sup 1 /\{(1-1 / r(x)) q(x)\}}\right)^{1 / q(x)} \\
& \left.\left.\times\left\{\int_{a}^{x} t^{1 /\{(1-1 / r(t)) q(t)\}} f^{p(t)}(t) d t\right\}^{1 / p(x)}\right\}^{p(x)} d x\right] \\
& \leq \alpha^{\sup p(x)}\left\{\inf \left\{\left(1-\frac{1}{r(x)}\right) q x\right\}\right\}^{\sup \{p(x) / q(x)\}} \\
& \times \int_{a}^{b} x^{-(1-1 / r(x)) p(x)}\left(x^{\sup 1 /\{(1-1 / r(x)) q(x)\}}-a^{\sup 1 /\{(1-1 / r(x)) q(x)\}}\right)^{p(x) / q(x)} \\
& \times \int_{a}^{x} t^{1 / q(t)} f^{p(t)}(t) d t d x \\
& \leq \alpha^{\sup p(t)}\left\{\inf \left\{\left(1-\frac{1}{r(t)}\right) q(t)\right\}\right\}^{\sup p(t) / q(t)} \\
& \times \int_{a}^{b}\left\{\int_{t}^{b} x^{-(1-1 / r(x)) p(x)+\sup \left(p(x) /(1-1 / r(x)) q(x)^{2}\right)}\right. \\
& \left.\times\left(1-\left(\frac{a}{x}\right)^{\sup (1 /(1-1 / r(x)) q(x))}\right)^{p(x) / q(x)} d x\right\} \\
& \times t^{1 /\{(1-1 / r(t)) q(t)\}} f^{p(t)}(t) d t \leq \alpha^{M}\left\{\inf \left\{\left(1-\frac{1}{r(t)}\right) q(t)\right\}\right\}^{\sup (p(t)-1)}
\end{aligned}
$$


S. K. Sunanda et al. 11

$$
\begin{aligned}
& \times \int_{a}^{b}\left\{\int_{t}^{b} x^{-1-1 /\{(1-1 / r(x)) q(x)\}}\left(1-\left(\frac{a}{x}\right)^{\sup (1 /(1-1 / r(x)) q(x))}\right)^{p(x) / q(x)} d x\right\} \\
& \times t^{1 /\{(1-1 / r(t)) q(t)\}} f^{p(t)}(t) d t \\
\leq & \alpha^{M}\left\{\inf \left\{\left(1-\frac{1}{r(t)}\right) q(t)\right\}\right\}^{\sup \{p(t)(1-1 / r(t))\}} \\
& \times\left[1-\left(\frac{a}{b}\right)^{\sup (1 /(1-1 / r(t)) q(t))}\right]^{\sup \{p(t)(1-1 / r(t))-1\}} \\
& \times\left[1-\left(\frac{t}{b}\right)^{\sup (1 /(1-1 / r(t)) q(t))}\right] f^{p(t)}(t) d t \\
\leq & \alpha^{M}\left\{\inf \left\{\left(1-\frac{1}{r(t)}\right) q(t)\right\}\right\}^{N}\left[1-\left(\frac{a}{b}\right)^{\sup (1 /(1-1 / r(t)) q(t))}\right]^{N} \int_{a}^{b} f^{p(t)}(t) d t .
\end{aligned}
$$

This completes the proof of our theorem.

Theorem 3.4. Let $a>0$, for all $x \in(a, \infty), p(x)>1, p(x)^{-1}+q(x)^{-1}=1-r(x)^{-1}, f \geq 0$, $r(x)>1$, and $0<\int_{a}^{\infty} f^{p(t)}(t) d t<\infty$. Then

$$
\begin{aligned}
\int_{a}^{\infty} & \left(\frac{1}{x^{(1-1 / r(x))}} \int_{a}^{x} f(t) d t\right)^{p(x)} d x \\
& <\alpha^{M}\left\{\inf \left\{\left(1-\frac{1}{r(t)}\right) q(t)\right\}\right\}^{N} \int_{a}^{\infty}\left[1-\theta_{N}(t)\right] f^{p(t)}(t) d t
\end{aligned}
$$

where $\theta_{N}(t)=(1 / N)\left[\sum_{k=1}^{\infty}(\Gamma(N+1) / \Gamma(k+2) \Gamma(N-k))(-1)^{k-1}(a / t)^{k /(1-1 / r(t)) \inf q(t)}\right]$, for $t>a, M=\sup p(t), \alpha=\sup p(t)^{-1}+\sup q(t)^{-1}+\sup r(t)^{-1}$, and $N=\sup p(t)(1-1 / r(t))$.

Proof. In view of inequalities (3.3) and (3.5), we find

$$
\begin{aligned}
\int_{a}^{\infty}( & \left.\frac{1}{x^{1-1 / r(x)}} \int_{a}^{x} f(t) d t\right)^{p(x)} d x \\
< & \alpha^{\sup p(x)}\left\{\inf \left(1-\frac{1}{r(x)}\right) q(x)\right\}^{\sup \{p(x) / q(x)\}} \\
& \quad \times \int_{a}^{\infty} x^{-(1-1 / r(x)) p(x)}\left(x^{\sup 1 /(1-1 / r(x)) q(x)}-a^{\sup (1 /(1-1 / r(x)) q(x))}\right)^{p(x) / q(x)} \\
& \times \int_{a}^{\infty} t^{1 /(1-1 / r(t)) q(t)} f^{p(t)}(t) d t d x \\
\leq & \alpha^{\sup p(t)}\left\{\inf \left(1-\frac{1}{r(t)}\right) q(t)\right\}^{\sup \{p(t)-p(t) / r(t)-1\}}
\end{aligned}
$$


12 Some new generalizations of Hardy's integral inequality

$$
\begin{aligned}
& \times \int_{a}^{\infty}\left\{\int_{t}^{\infty} x^{-(1-1 / r(x)) p(x)+p(x) /(1-1 / r(x)) q(x)^{2}}\right. \\
& \left.\times\left(1-\left(\frac{a}{x}\right)^{\sup (1 /(1-1 / r(x)) q(x))}\right)^{p(x) / q(x)} d x\right\} t^{1 /(1-1 / r(t)) q(t)} f^{p(t)}(t) d t \\
& \leq \alpha^{M}\left\{\inf \left(1-\frac{1}{r(t)}\right) q(t)\right\}^{\sup \{p(t)(1-1 / r(t)\}} \\
& \times \int_{a}^{\infty}\left[\int_{t}^{\infty}\left(1-\left(\frac{a}{x}\right)^{\sup 1 /(1-1 / r(x)) q(x)}\right)^{\sup p(x) / q(x)}\right. \\
& \left.\times d\left(1-\left(\frac{a}{x}\right)^{\sup 1 /(1-1 / r(x)) q(x)}\right)\right]\left(\frac{t}{a}\right)^{\sup 1 /(1-1 / r(t)) q(t)} f^{p(t)}(t) d t \\
& \leq \alpha^{M}\left\{\inf \left(1-\frac{1}{r(t)}\right) q(t)\right\}^{\sup \{p(t)(1-1 / r(t))\}} \\
& \times \int_{a}^{\infty} \frac{1}{\sup (1-1 / r(t)) p(t)}\left\{1-\left[1-\left(\frac{a}{t}\right)^{\sup 1 /(1-1 / r(t)) q(t)}\right]^{\sup (1-1 / r(t)) p(t)}\right\} \\
& \times\left(\frac{t}{a}\right)^{\sup 1 /(1-1 / r(t)) q(t)} f^{p(t)}(t) d t \\
& =\alpha^{M}\left\{\inf \left(1-\frac{1}{r(t)}\right) q(t)\right\}^{N} \int_{a}^{\infty}\left[1-\theta_{N}(t)\right] f^{p(t)}(t) d t,
\end{aligned}
$$

where $N=\sup 1 /(1-1 / r(t)) p(t)>1$,

$$
\begin{gathered}
\theta_{N}(t)=1-\frac{1}{N}\left\{1-\left[1-\left(\frac{a}{t}\right)^{\sup (1 /(1-1 / r(t)) q(t))}\right]^{N}\right\}\left(\frac{t}{a}\right)^{\sup (1 /(1-1 / r(t)) q(t))}, \quad t>a>0, \\
\theta_{N}(a)=\sup \frac{1}{(1-1 / r(t))} q(t),
\end{gathered}
$$

since

$$
\begin{gathered}
{\left[1-\left(\frac{a}{t}\right)^{\sup 1 /(1-1 / r(t)) q(t)}\right]^{N}=\sum_{k=0}^{\infty} \frac{\Gamma(N+1)}{\Gamma(k+1) \Gamma(N-k+1)}(-1)^{k}\left(\frac{a}{t}\right)^{k / \inf 1 /(1-1 / r(t)) q(t)},} \\
\theta_{N}(t)=\frac{1}{N} \sum_{k=0}^{\infty} \frac{\Gamma(N+1)}{\Gamma(k+2) \Gamma(N-k)}(-1)^{k-1}\left(\frac{a}{t}\right)^{k / \inf (1-1 / r(t)) q(t)}, \quad t>a>0 .
\end{gathered}
$$

This completes the proof. 
Note. When $t>a>0$, by Bernoulli's inequality (see [2, Chapter 2.4]), we obtain

$$
\begin{gathered}
1-N\left(\frac{a}{t}\right)^{\sup 1 /(1-1 / r(t)) q(t)}<\left[1-\left(\frac{a}{t}\right)^{\sup 1 /(1-1 / r(t)) q(t)}\right]^{N}, \\
\theta_{N}(t)>1-\frac{1}{N}\left[1-\left\{1-N\left(\frac{a}{t}\right)^{\sup 1 /(1-1 / r(t)) q(t)}\right\}\right]\left(\frac{t}{a}\right)^{\sup 1 /(1-1 / r(t)) q(t)}=0 .
\end{gathered}
$$

\section{Applications}

Theorem 3.5. Let $0<b \leq \infty$, for all $x \in(0, \infty), s \geq N>1, p(x)^{-1}+q(x)^{-1}=1-r(x)^{-1}$, $f \geq 0, r(x)>1$, and $0<\int_{0}^{b} x^{-s+N} f^{p(x)}(x)<\infty$. Then

(i) for $b \in(0, \infty)$,

$$
\begin{aligned}
\int_{0}^{b} x^{-s} & \left(\int_{0}^{x} f(t) d t\right)^{p(x)} d x \\
< & \frac{\alpha^{M}\{\inf (1-1 / r(t)) q(t)\}^{N}}{\{\inf (1-1 / r(t)) q(t)\}(s-N)+1} \int_{0}^{b}\left[1-\left(\frac{t}{b}\right)^{\{s-N+\inf 1 /(1-1 / r(t)) q(t)\}}\right] \\
& \times t^{-s+(1-1 / r(t)) p(t)} f^{p(t)}(t) d t
\end{aligned}
$$

(ii) for $b=\infty$,

$$
\begin{aligned}
& \int_{0}^{\infty} x^{-s}\left(\int_{0}^{x} f(t) d t\right)^{p(x)} d x<\frac{\alpha^{M}\{\inf (1-1 / r(t)) q(t)\}^{N}}{\{\inf (1-1 / r(t)) q(t)\}(s-N)+1} \int_{0}^{\infty} t^{-s+N} f^{p(t)}(t) d t, \\
& M=\sup p(t), \quad \alpha=\sup p(t)^{-1}+\sup q(t)^{-1}+\sup r(t), \quad N=\sup p(t)\left(1-\frac{1}{r(t)}\right) .
\end{aligned}
$$

Proof. For case (i), $b \in(0, \infty)$, we use (3.1) to obtain

$$
\begin{aligned}
\int_{0}^{b} x^{-r} & \left(\int_{0}^{x} f(t) d t\right)^{p(x)} d x \\
< & \alpha^{M}\left\{\inf \left(1-\frac{1}{r(x)}\right) q(x)\right\}^{\sup p(x) / q(x)} \int_{0}^{b} x^{-s+\sup \{1 /((1-1 / r(x)) q(x) 2)\}} \\
& \times \int_{0}^{x} t^{1 /(1-1 / r(t)) q(t)} f^{p(t)}(t) d t d x \\
\leq & \alpha^{M}\left\{\inf \left(1-\frac{1}{r(t)}\right) q(t)\right\}^{\sup \{p(t) / q(t)\}} \\
& \times \int_{0}^{b}\left(\int_{t}^{b} x^{-s+\sup \{1 /(1-1 / r(x)) p(x)-1 /(1-1 / r(x)) q(x)-1\}} d x\right) t^{1 /(1-1 / r(t)) q(t)} f^{p(t)}(t) d t
\end{aligned}
$$


14 Some new generalizations of Hardy's integral inequality

$$
\begin{aligned}
\leq & \frac{\alpha^{M}\{\inf (1-1 / r(t)) q(t)\}^{\sup (1-1 / r(t)) p(t)-1}}{-s+\sup \{(1-1 / r(t)) p(t)-1 /(1-1 / r(t)) q(t)\}} \\
& \times \int_{0}^{b}\left(b^{-s+\sup \{(1-1 / r(t)) p(t)-1 /(1-1 / r(t)) q(t)\}}-t^{-s+\sup \{(1-1 / r(t)) p(t)-1 /(1-1 / r(t)) q(t)\}}\right) \\
& \times t^{1 /(1-1 / r(t)) q(t)} f^{p(t)}(t) d t \\
\leq & \frac{\alpha^{M}\{\inf (1-1 / r(t)) q(t)\}^{N}}{\{\inf (1-1 / r(t)) q(t)\}(s-N)+1} \int_{0}^{b}\left[1-\left(\frac{t}{b}\right)^{s-N+\inf \{(1-1 / r(t)) q(t)\}}\right] \\
& \times t^{-s+\sup (1-1 / r(t)) p(t)} f^{p(t)}(t) d t .
\end{aligned}
$$

For case (ii), $b=\infty$, we use (3.1) to find

$$
\begin{aligned}
\int_{0}^{\infty} x^{-s} & \left(\int_{0}^{x} f(t) d t\right)^{p(x)} d x \\
< & \alpha^{M}\left\{\inf \left(1-\frac{1}{r(x)}\right) q(x)\right\}^{\sup p(x) / q(x)} \int_{0}^{\infty} x^{-s+\sup \left\{1 /(1-1 / r(x)) q(x)^{2}\right\}} \\
& \times \int_{0}^{x} t^{1 /(1-1 / r(t)) q(t)} f^{p(t)}(t) d t d x \\
= & \alpha^{M}\left\{\inf \left(1-\frac{1}{r(t)}\right) q(t)\right\}^{\sup p(t) / q(t)} \\
& \times \int_{0}^{\infty}\left(\int_{t}^{\infty} x^{-s+\sup \{1 /(1-1 / r(x)) p(x)-1 /(1-1 / r(x)) q(x)-1\}} d x\right) \\
& \times t^{1 /(1-1 / r(t)) q(t)} f^{p(t)}(t) d t \\
= & \frac{\alpha^{M}\{\inf (1-1 / r(t)) q(t)\}^{N}}{\{\inf (1-1 / r(t)) q(t)\}(s-N)+1} \int_{0}^{\infty} t^{-s+N} f^{p(t)}(t) d t .
\end{aligned}
$$

Remark 3.6. (a) If $p(x), q(x)$, and $r(x)$ are constants in Lemmas 3.1 and 3.2 and Theorems 3.3 and 3.4, then our results reduce to the corresponding Lemmas 2.1 and 2.2 and Theorems 2.4 and 2.5 obtained in [3].

(b) If we take $r(x) \rightarrow \infty$ in Theorems 3.3 and 3.4, then it reduces to corresponding Theorems 2.4 and 2.5 .

(c) In the limits $a \rightarrow 0, b \rightarrow \infty, r(x) \rightarrow \infty, p(x)$ and $q(x)$ constants, (3.5) reduces to (1.1). Hence (3.5) is the generalization of (1.1).

\section{Acknowledgments}

The authors wish to thank the referees and Professor Lokenath Debnath, IJMMS Managing Editor for their valuable suggestions which improved the presentation of the paper. 


\section{References}

[1] Y. Bicheng, Z. Zhuohua, and L. Debnath, On new generalizations of Hardy's integral inequality, Journal of Mathematical Analysis and Applications 217 (1998), no. 1, 321-327.

[2] G. H. Hardy, J. E. Littlewood, and G. Pólya, Inequalities, Cambridge University Press, Massachusetts, 1952.

[3] J. A. Oguntuase and C. O. Imoru, New generalizations of Hardy's integral inequality, Journal of Mathematical Analysis and Applications 241 (2000), no. 1, 73-82.

S. K. Sunanda: Department of Mathematics, Indian Institute of Technology, Kharagpur, Kharagpur 721 302, India

E-mail address: sunanda@maths.iitkgp.ernet.in

C. Nahak: Department of Mathematics, Indian Institute of Technology, Kharagpur, Kharagpur 721 302, India

E-mail address:cnahak@maths.iitkgp.ernet.in

S. Nanda: Department of Mathematics, Indian Institute of Technology, Kharagpur, Kharagpur 721 302, India

Current address: North Orissa University, Baripada, Distt. Mayurbhanj, Orissa 757003, India E-mail address: snanda@maths.iitkgp.ernet.in 


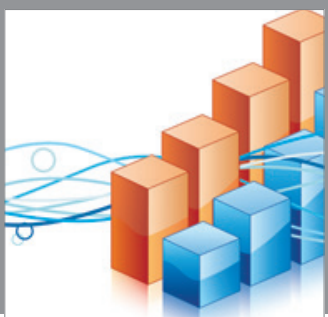

Advances in

Operations Research

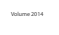

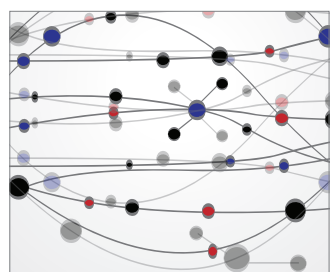

\section{The Scientific} World Journal
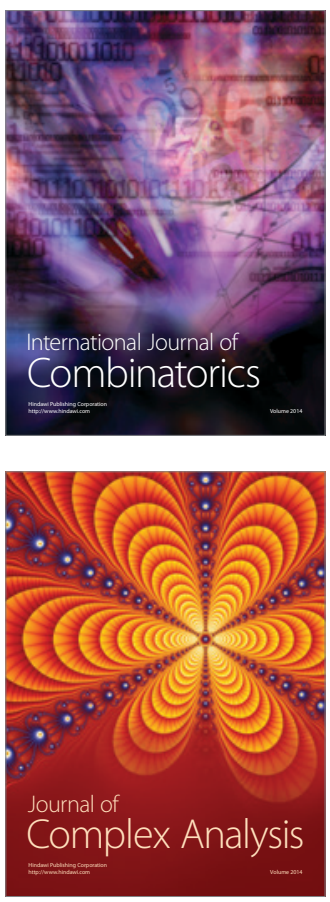

International Journal of

Mathematics and

Mathematical

Sciences
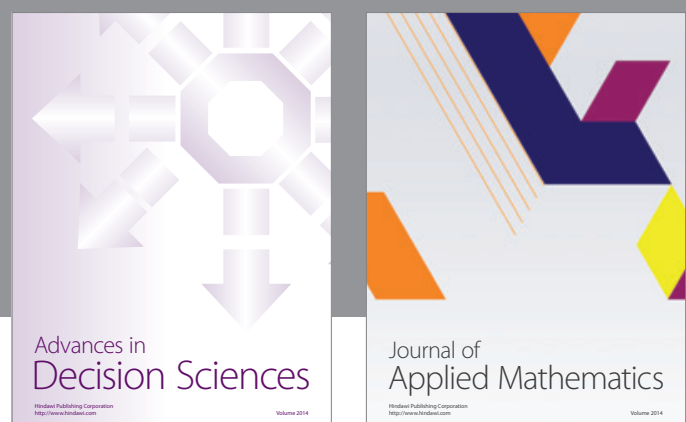

Journal of

Applied Mathematics
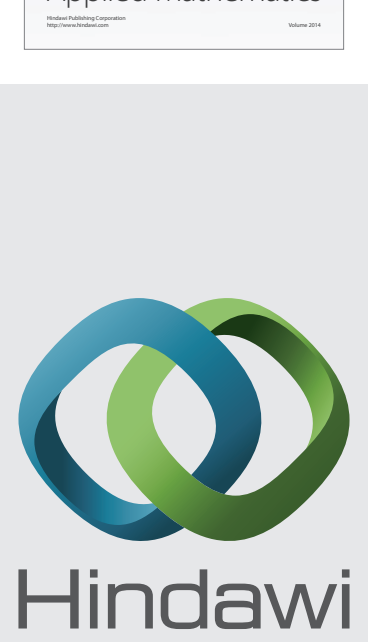

Submit your manuscripts at http://www.hindawi.com
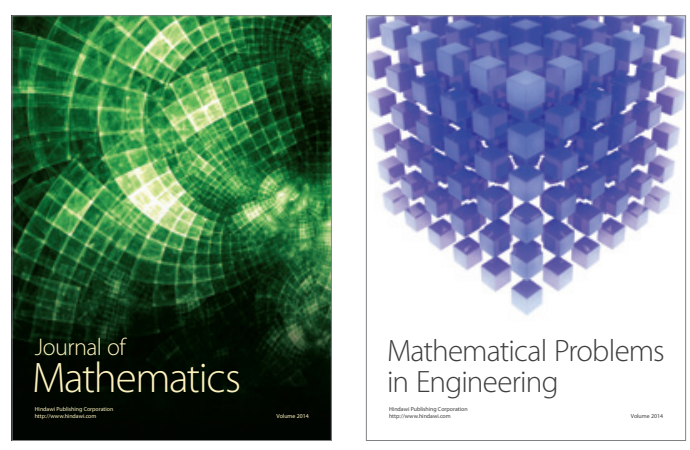

Mathematical Problems in Engineering
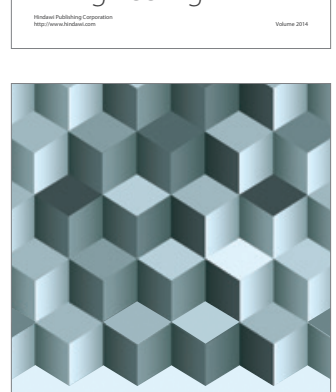

Journal of

Function Spaces
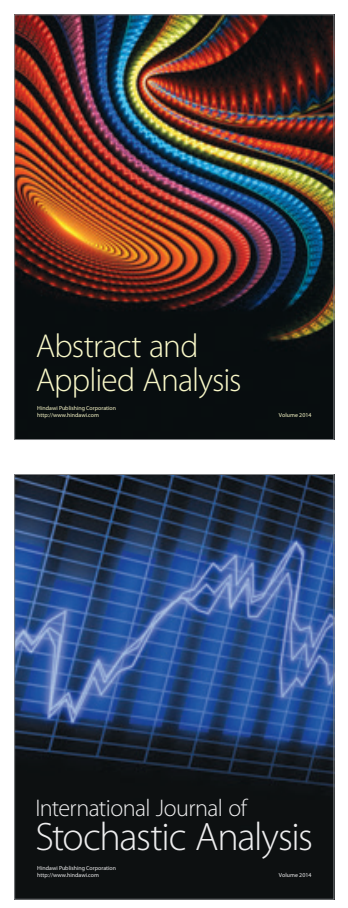

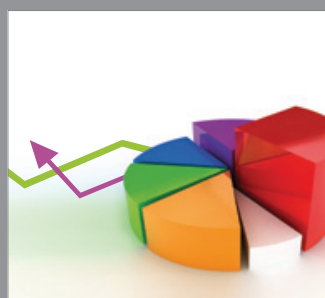

ournal of

Probability and Statistics

Promensencen
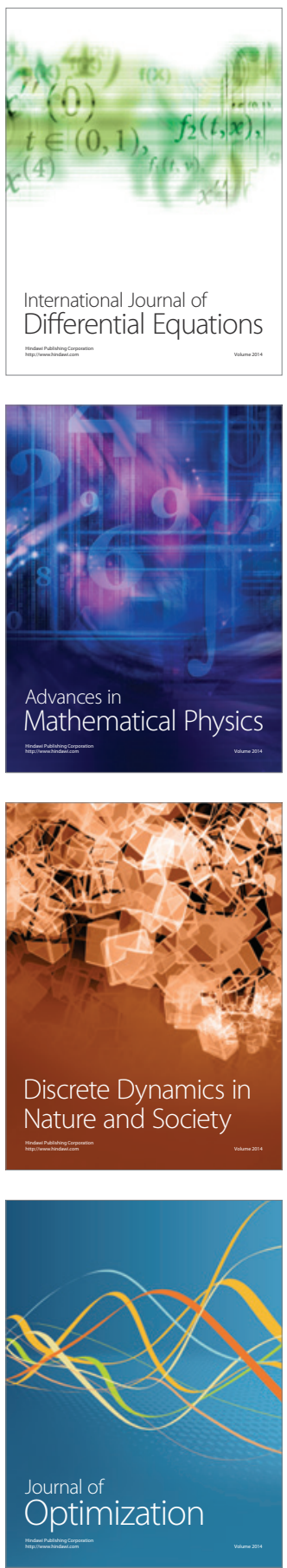